\title{
Behavioral changes in group-housed dairy calves infected with Mannheimia haemolytica
}

\author{
C. L. Hixson, ${ }^{*}$ P. D. Krawczel, $†$ J. M. Caldwell,‡ and E. K. Miller-Cushon*1 \\ *Department of Animal Sciences, University of Florida, Gainesville 32611 \\ †Department of Animal Science, and \\ $\ddagger$ College of Veterinary Medicine, University of Tennessee, Knoxville 37996
}

\section{ABSTRACT}

Monitoring sickness behavior may improve identification, management, and welfare of sick animals. The objective of this study was to characterize components of sickness behavior in group-housed dairy calves, using an experimental disease challenge model with Mannheimia haemolytica (MH). Holstein bull calves (aged 3-7 weeks; $58.0 \pm 12.0 \mathrm{~kg}$ of body weight) were group-housed based on age and body weight in sand-bedded pens (6 calves/pen, $6.6 \mathrm{~m}^{2} /$ calf) and provided pasteurized waste milk $(8 \mathrm{~L} / \mathrm{d}) 2 \times / \mathrm{d}$ and grain concentrate ad libitum. Within group, calves were randomly assigned to 1 of 2 treatments: (1) inoculation at the tracheal bifurcation with $3 \times 10^{9}$ cfu of $\mathrm{MH}$ suspended in $5 \mathrm{~mL}$ of sterile phosphate buffered saline (PBS) followed by a $120 \mathrm{~mL}$ wash PBS (MH; $\mathrm{n}=12,3 /$ pen), or (2) inoculation with $5 \mathrm{~mL}+120 \mathrm{~mL}$ of sterile PBS only (control; $\mathrm{n}=12,3 /$ pen). Rectal temperature and health scores were collected from d 0 to +6 of the challenge. A range of behaviors, including feeding patterns and social interactions, were recorded from video from d 0 to +2 . The challenge model resulted in calves experiencing a mild disease state: rectal temperatures of $\mathrm{MH}$ calves were elevated throughout the challenge compared with control calves, peaking at $12 \mathrm{~h}$ postinoculation (39.2 vs. $38.9^{\circ} \mathrm{C}$; standard error $\left.=0.14\right)$. Many behavioral responses were subject to treatment by day effects, with calves generally becoming less active following inoculation with $\mathrm{MH}$ and then returning to baseline. Affected behaviors surrounding feeding included milk feeding time, frequency of competitive displacements, and concentrate feeding time. Lying time was similarly subject to treatment by day effects, and $\mathrm{MH}$ calves also spent more time lying on their left side compared with their right $(604$ vs. $471 \mathrm{~min} / \mathrm{h}$; standard error $=32$ ), whereas control calves expressed no laterality.

\footnotetext{
Received March 26, 2018.

Accepted July 22, 2018.

${ }^{1}$ Corresponding author: emillerc@ufl.edu
}

Duration of social lying did not differ, but frequency of social lying bouts decreased in $\mathrm{MH}$ calves following inoculation ( 0.44 vs. 0.75 bouts/h; standard error = $0.04)$. Social grooming was initiated less by $\mathrm{MH}$ calves $(0.78$ vs. $1.96 \mathrm{~min} / \mathrm{h}$; standard error $=0.38)$, but they tended to receive more social grooming for a greater duration of time (1.59 vs. $1.25 \mathrm{~min} / \mathrm{h}$; standard error $=$ 0.13). Overall, we found that infected calves exhibited reduced grooming, feeding, and social interactions, suggesting that these behavioral changes may be useful indicators of early stages of respiratory disease.

Key words: dairy calf, bovine respiratory disease, social behavior, feeding behavior

\section{INTRODUCTION}

Morbidity and mortality of dairy calves are major concerns from economic and animal welfare viewpoints. Bovine respiratory disease is the leading cause of death in dairy calves, affecting a reported $12.4 \%$ of preweaned calves and causing $22.5 \%$ of calf deaths (USDA, 2008). Successful identification of respiratory disease remains difficult, as the criteria is subjective and inconsistent, resulting in failure to identify and treat a sick animal as well as unnecessary treatment (Kelly and Janzen, 1986; Love et al., 2014).

Behavioral indicators of disease may assist with prompt identification and treatment of sick animals. Animals respond to developing disease with behavioral changes, which may be a coordinated strategy to conserve energy and mount an immune response (Hart, 1988). Previous research has identified changes in feeding behavior associated with disease; for example, morbid steers spend less time at the feed bunk and have fewer meals than healthy animals (Sowell et al., 1999). In dairy calves, the response of feeding behavior to disease appears to depend on feeding motivation, as sick calves will consume less when milk is available ad libitum but not when milk allowance is restricted and feeding motivation is high (Borderas et al., 2009). Thus, better understanding of the full expression of sickness behavior within the context of different management 
environments is needed to effectively use behavioral indicators to assist with early identification of disease.

On-farm approaches to rearing dairy calves are currently varied and behavior of calves is highly subject to management and housing factors. In particular, it is becoming more common to house dairy calves in groups, which provides benefits including greater solid feed intake and reduced stress around weaning (De Paula Vieira et al., 2010), and supports social development (Veissier et al., 1994; Duve and Jensen, 2012). However, housing calves in large groups may increase the risk of respiratory disease compared with individual housing or smaller group-housing (Svensson et al., 2003; Svensson and Liberg, 2006) and could pose challenges for identifying disease, as calves may receive less individual monitoring at feeding. Understanding behavioral changes that may be associated with disease in a social environment, such as social withdrawal or changes in daily activity patterns, may provide insight into improved identification and management strategies for sick group-housed calves. Weary et al. (2009) suggested that sick animals are likely to spend less time performing behaviors that are less critical for survival, including social behavior, grooming, and exploration, suggesting these may be useful indicators of developing disease.

Characterizing sickness behavior often relies on bouts of naturally occurring disease. However, those studies are often limited by the need to monitor a large number of calves to obtain a sufficient number of disease cases. Consequently, behavioral data collection may be restricted to few outcomes, such as measures which can be collected by automated milk feeders (Svensson and Jensen, 2007; Borderas et al., 2009). Additionally, different pathogens can have result in different behavioral changes (Weary et al., 2009), such that untangling behaviors predictive of specific diseases is difficult. Experimental disease challenge models can be a useful approach to evaluate a wide range of behavioral responses in a controlled setting with fewer experimental animals. Borderas et al. (2008) investigated a low-dose LPS challenge model in dairy calves and noted some behavioral changes, including reduced grooming. However, this challenge model was limited by the short duration of the effect, as rectal temperature peaked within $4 \mathrm{~h}$ of induction (Borderas et al., 2008). Although experimental disease challenge models may have the limitation of not accounting for physiological processes associated with the progression of naturally occurring disease (Weary et al., 2009), some pathogen challenge models may have potential to produce a sickness response that mirrors naturally occurring disease more closely. Some recent studies in both beef and dairy calves have involved challenges of Mannheimia haemolytica, which is considered the predominant bacterial pathogen associated with bovine respiratory disease (Griffin et al., 2010). Beef calves challenged with $M$. haemolytica by inoculation in the tracheal bronchus exhibited changes in behavior, including reduced time spent at the feeder and increased time lying, for 24 to $48 \mathrm{~h}$ postchallenge (Theurer et al., 2013). Dairy calves challenged with $M$. haemolytica exhibited slight changes in lying behavior and increased rectal temperature, peaking at $12 \mathrm{~h}$ postinoculation (Eberhart et al., 2017), suggesting this model may be an effective approach to evaluate mild sickness behavior in a controlled setting conducive to intensive behavioral observation.

Despite increasing prevalence of social housing systems, understanding of the range of behavioral changes associated with disease in group-housed calves is limited. Our objective was to characterize the components of sickness behavior in group-housed dairy calves using an experimental disease challenge model. We hypothesized that sickness behavior would be characterized by decreased time spent feeding, standing, and engaged in active behaviors, such as grooming. Group-housed calves exhibit behaviors not available to individually housed calves, including social interactions surrounding feeding, social grooming, and lying in proximity to other calves (Færevik et al., 2007; Miller-Cushon et al., 2014), which we predicted would be altered in response to infection. Finally, we predicted that sickness behavior would include changes in exploratory behavior and response to novelty, as assessed using a novel object test.

\section{MATERIALS AND METHODS}

\section{Animals and Management}

Twenty-four Holstein bull calves were born consecutively and enrolled on this trial at the University of Florida Dairy Research Unit (Hague). Only male calves were used due to facility limitations in providing heifers for this research. Calves were fed $6 \mathrm{~L}$ of quality-controlled colostrum in 2 feedings $(3 \mathrm{~L}$ within $4 \mathrm{~h}$, and $3 \mathrm{~L}$ within $12 \mathrm{~h}$ ), individually housed from birth until 30.9 $\pm 1.8 \mathrm{~d}$ of age in wire mesh pens $(0.9 \times 1.8 \mathrm{~m}$; width $\times$ depth), and then group-housed based on age (6 calves/ pen; $6.6 \mathrm{~m}^{2} /$ calf), following standard operating procedure for group-housed calves at this facility. Age range within each group was $7.5 \pm 3.3 \mathrm{~d}$ (mean \pm SD). All pens were bedded with sand, which was cleaned daily and replaced as needed. Pens were located under an open-sided building to allow for natural ventilation and protection from rain. All management and experiment procedures were approved by the Institutional Animal Care and Use Committee (\#201408643). 
Calves were provided pasteurized waste milk mixed with a powdered enhancer (Pasteurized Milk Balancer Protein-Blend, Purina Animal Nutrition LLC, Shoreview, MN), provided at a rate of $8 \mathrm{~L} / \mathrm{d}$ via 2 daily meals (0600 and $1700 \mathrm{~h})$. Milk was offered via teats (Peach Teats, JDJ Solutions, Homer, NY) mounted on one side of the pen, connected externally to milk buckets through tubes fitted with 1-way valves. After calves had finished consuming milk and were no longer present in the feeding area, teats were removed, disinfected, and replaced to provide opportunity for nonnutritive sucking. Grain concentrate was provided ad libitum via buckets mounted to the side of the pen (20\% CP; Ampli-Calf Warm Weather Starter, Purina Animal Purina Nutrition LLC, Shoreview, MN), and water was provided ad libitum and replaced daily.

\section{Experimental Design}

Within each group, calves were blocked into pairs by age and BW, and randomly assigned (using the random group number generator in Microsoft Office Excel, Microsoft, Redmond, WA) to 1 of 2 treatments, implemented across all calves on the same calendar day, when the mean calf age (across all 4 pens) was 35.9 $\pm 8.8 \mathrm{~d}$ : (1) respiratory disease challenge induced by endoscopic inoculation with Mannheimia haemolytica (MH) or (2) inoculation with sterile saline only as control $(\mathbf{C O N})$. At the time of inoculation, the specific age range in each pen was (mean $\pm \mathrm{SD}$ ): $46.5 \pm 1.52 \mathrm{~d}$ in pen $1,41.0 \pm 3.2 \mathrm{~d}$ in pen $2,29.7 \pm 5.1 \mathrm{~d}$ in pen 3 , and $26.5 \pm 1.87 \mathrm{~d}$ in pen 4 .

Sample size was decided based on data obtained from this challenge model in 8-wk-old Holstein dairy calves (Eberhart et al., 2017). Based on reported differences in lying behavior in response to the challenge, it was determined that a sample size of 8 would be sufficient to determine differences in activity, using a level of significance of 0.05 and $80 \%$ power. However, given the lack of previous data for certain behavioral responses of interest in the present study, such as social behavior, the sample size was increased to 12 calves/treatment to increase power to detect differences in behavioral responses which may be subject to greater individual variability than lying time.

Throughout the experiment, biosecurity measures (protective clothing and boot covers for staff) were in place and animals were physically isolated from the rest of the herd. At $14 \mathrm{~d}$ postinoculation, calves received final veterinary examinations, were treated with an antimicrobial [40 mg $/ \mathrm{kg}$ subcutaneous injection, Nuflor (Florfenicol), Merck Animal Health, Kenilworth, NJ], and were subsequently managed as per standard farm operating procedure for bull calves.

\section{Disease Challenge}

On d 0, endoscopic inoculation, based on the model described by Eberhart et al. (2017), was performed approximately 6 to $10 \mathrm{~cm}$ proximal to the tracheal bifurcation, the point at which the trachea ends and divides into 2 principal bronchi. For the procedure, calves remained in the pen and were haltered and restrained with the head elevated. The external nasal area was cleaned and a $5.9 \mathrm{~mm}$ endoscope with $2 \mathrm{~mm}$ biopsy channel was introduced into the nasal passage. The endoscope was passed through the nasopharyngeal region and subsequently through the laryngeal folds into the trachea. The endoscope was passed into smaller airways until the tracheal bifurcation was identified. A 140-cmlong, 1.95-mm-diameter polyurethane catheter was passed through the endoscopic biopsy channel and the inoculum ( $\mathrm{MH}$ calves received $3 \times 10^{9}$ cfu of M. haemolytica suspended in $5 \mathrm{~mL}$ of PBS, and control calves received $5 \mathrm{~mL}$ of sterile $\mathrm{PBS}$ ) was delivered through the catheter, followed by a $120-\mathrm{mL}$ wash with sterile PBS. The catheter was then slowly withdrawn and the restraint relieved. All inoculations were performed by a single veterinarian experienced in this experimental procedure.

\section{Health Data Collection}

Baseline health scores were collected weekly for all calves following birth to ensure no calves that were clinically sick before the challenge were included in the experiment. Health status of all calves was evaluated using noninvasive assessments of health based on presence of cough, nasal or ocular discharge, ear droop, and rectal temperature (using the Calf Health Scoring Chart from the University of Wisconsin-Madison; available at http://www.vetmed.wisc.edu/dms/fapm/ fapmtools/8calf/calf_respiratory_scoring_chart.pdf). Health scores were collected by a single observer that was trained by a veterinarian before the start of the experiment, and intra-observer reliability was verified by repeated scoring of calves during early weeks of the experiment (100\% agreement was noted). To prevent the possibility of bias, information about calf treatment assignments was not available during health scoring. Two days before the challenge, health scores were recorded twice daily, and continued for $6 \mathrm{~d}$ following the challenge.

\section{Behavioral Observation}

The behavior of all calves was recorded using digital video cameras (Foscam F1990EP, Foscam Digital Technologies, Houston, TX) mounted above each pen, 
connected to a server running video recording software (Blue Iris Video Security Software, Hyland Software, $\mathrm{OH})$. To characterize a range of behaviors (Table 1), calves were observed during daylight hours (0500 to $2000 \mathrm{~h}$ ) for $3 \mathrm{~d}$ beginning at time of inoculation (d 0 to +2 ). Individual calves were identified by markings and behaviors were recorded continuously by a single observer using event-logging software (Behavioral Observation Research Interactive Software; Friard and Gamba, 2016). The observer was blind to treatment during video observation, and intra-observer reliability, as measured with regression, was high $\left(\mathrm{R}^{2} \geq 86 \%\right.$ for all behaviors). Behavioral data were calculated as hourly means, for behavior that occurred throughout the day, or per meal, for milk feeding behavior, and summarized across each observation period. To assess directionality of social grooming behavior, we calculated the ratio of initiated to received social grooming (duration of social grooming performed divided by duration of social grooming received). Similarly, to assess directionality of involvement in competitive interactions, we calculated the ratio of initiated to received displacements for access to a teat providing milk [frequency of displacements initiated (focal calf was actor) divided by frequency of received displacements].

In addition to behaviors recorded from video, calves were fitted with accelerometers (HOBO Pendant G Data Logger, Onset Computer Corp., Pocasset, MA) to record standing and lying behavior, as validated in calves by Bonk et al. (2013). Loggers were secured to a rear leg using bandage tape (Vetrap Bandaging Tape, 3M, Bracknell, UK) as per the protocol developed by UBC AWP (2013). Loggers were attached 12 $\mathrm{d}$ before inoculation and set to record for $7 \mathrm{~d}$ following inoculation. Daily total lying time, time spent lying on either side of the body (lying laterality), and lying bout frequency were calculated from collected data. Due to logger malfunction, data were not available for 2 calves (MH treatment) for the duration of the study.

\section{Novel Object Test}

To assess differences in response to novelty and exploratory behavior following infection, we conducted a novel object test on $d+2$ following treatment, adapted from methods developed by Cramer and Stanton (2015). At $1000 \mathrm{~h}$, a red and white colored ball (36 $\mathrm{cm}$ in diameter), was placed in the center of the home group pen. Behavioral observations of individual calves were made for $10 \mathrm{~min}$ following introduction of the ball. The duration of time within 1 head length of the ball (described as "almost touching"), latency to contact the ball, and duration in contact with the ball were recorded.

\section{Statistical Analyses}

All data were summarized by day. Prior to analysis, data were checked for normality using the UNIVARIATE procedure in SAS (SAS v.9.4, SAS Institute Inc., Cary, NC). To satisfy assumptions of normality, the following outcome variables were log-transformed: head-butting frequency and latency to touch the novel object.

To analyze the effect of treatment on health and behavior outcomes, data were analyzed using the MIXED procedure of SAS, treating day as a repeated measure. The model included the random effect of calf and the fixed effects of treatment, day, treatment by day interaction, and pen. In cases of significant treatment by day interactions, we tested for differences between treatments by day (using the SLICE command). Effects of pen reflected differences by age, as calves were grouped by age and assigned to treatment within group. The covariance structure with the lowest Schwarz Bayesian information criterion was selected for each variable (the heterogeneous autoregressive covariance structure was selected for most social and feeding behavior measures, and compound symmetry was selected for activity data). Lying laterality was analyzed using the above model plus split-plot effects to examine the effects of lying side and its interactions. Results from the novel object test were analyzed in a general linear mixed model including the random effects of calf within pen and the fixed effects of treatment and pen.

All values reported are least squares means. Significance was declared at $P<0.05$ and trends reported at $0.05 \leq P<0.10$.

\section{RESULTS}

The experimental disease challenge model resulted in elevated rectal temperatures and other signs of clinical disease in dairy calves. Rectal temperatures over the 7-d period following treatment were subject to a treatment by day interaction $\left(\mathrm{F}_{15,330}=1.90 ; P=0.02\right)$, with $\mathrm{MH}$ calves having a peak temperature at $12 \mathrm{~h}$ postinoculation $\left(40.1\right.$ vs. $39.1^{\circ} \mathrm{C}$; $\mathrm{MH}$ vs. $\mathrm{CON}$; $\mathrm{SE}=$ $\left.0.14, \mathrm{~F}_{1,330}=25.3 ; P<0.001\right)$ which gradually returned to baseline (Figure 1). Health scores were greater for $\mathrm{MH}$ calves overall (4.14 vs. $3.38 ; \mathrm{MH}$ vs. $\mathrm{CON}$; $\mathrm{SE}=$ $\left.0.22, \mathrm{~F}_{1,16}=5.87 ; P=0.028\right)$, reflecting evidence of clinical signs of disease, such as some presence of nasal discharge, in addition to elevated rectal temperatures.

During the 3 -d period of behavioral observation following the disease challenge, feeding behavior and social interactions around feeding were altered, especially immediately following inoculation. Milk feeding time was subject to a treatment by day interaction $\left(\mathrm{F}_{2,44}\right.$ 
Table 1. Ethogram used to describe behaviors of calves (35.9 $\pm 8.8 \mathrm{~d}$ of age) housed in groups (6 calves/pen) exposed to either an experimental disease challenge model (inoculation with Mannheimia haemolytica) or a sham procedure (inoculation with sterile saline), for a 3-d period following treatment

\begin{tabular}{ll}
\hline Behavior & Description \\
\hline Milk feeding & Mouth on the teat while milk is available \\
Nonnutritive sucking & Mouth on the teat when milk is not available \\
Concentrate feeding & Head lowered into feed bucket \\
Displacement & A calf (noted as reactor) is displaced at the feeding station following physical contact from another calf (noted as \\
& actor) \\
Social lying & Lying within one body length of another calf \\
Self-grooming & Licking or scratching any part of own body \\
Social grooming & Licking the head, neck, or body of another calf; identified as actor or recipient \\
Head butting & Pushing the head against the head or body of another calf; identified as actor or recipient \\
\hline
\end{tabular}

$=14.09 ; P<0.001)$, decreasing in $\mathrm{MH}$ calves immediately postinoculation and then increasing relative to CON over time (Figure 2a). The frequency of visits to the teat during milk meals were similarly subject to a treatment by day interaction $\left(\mathrm{F}_{2,44}=3.81 ; P=0.030\right.$; Figure $2 \mathrm{~b}$ ), but the duration of time spent suckling during each visit to the teat did not differ between treatments over time $(23.4$ vs. $18.7 \mathrm{~s} / \mathrm{visit}$ : MH vs. $\left.\mathrm{CON} ; \mathrm{SE}=9.1 ; \mathrm{F}_{2.34}=1.31 ; P=0.28\right)$. Inoculated calves were less competitive surrounding milk feeding; the frequency of initiated $\left(\mathrm{F}_{2,44}=7.79 ; P=0.0013\right)$ and received displacements $\left(\mathrm{F}_{2,44}=9.78 ; P<0.001\right)$ were both subject to treatment by day interactions, but the ratio of initiated to received displacements was not affected (0.79 vs. $0.74 ; \mathrm{SE}=0.10 ; \mathrm{MH}$ vs. $\mathrm{CON} ; \mathrm{F}_{2,44}$ $=1.26 ; P=0.29)$. The $\mathrm{MH}$ calves had less involvement in competition on d 0 but increased engagement in competitive interactions on the days following (Figure $2 c)$. The frequency of nonnutritive visits to the teat did not differ between treatments over time (2.22 vs. 1.95 visits/h; $\mathrm{MH}$ vs. $\mathrm{CON} ; \mathrm{SE}=0.31 ; \mathrm{F}_{2,44}=1.26 ; P=$ $0.30)$, but $\mathrm{MH}$ calves spent less time overall suckling during nonnutritive visits (5.19 vs. $6.70 \mathrm{~s} /$ visit; $\mathrm{MH}$ vs. $\left.\mathrm{CON} ; \mathrm{SE}=0.50 ; \mathrm{F}_{1,16}=4.61 ; P=0.047\right)$. Concentrate feeding time was subject to a treatment by day interaction $\left(\mathrm{F}_{2,44}=4.10 ; P=0.023\right)$, as well as a treatment

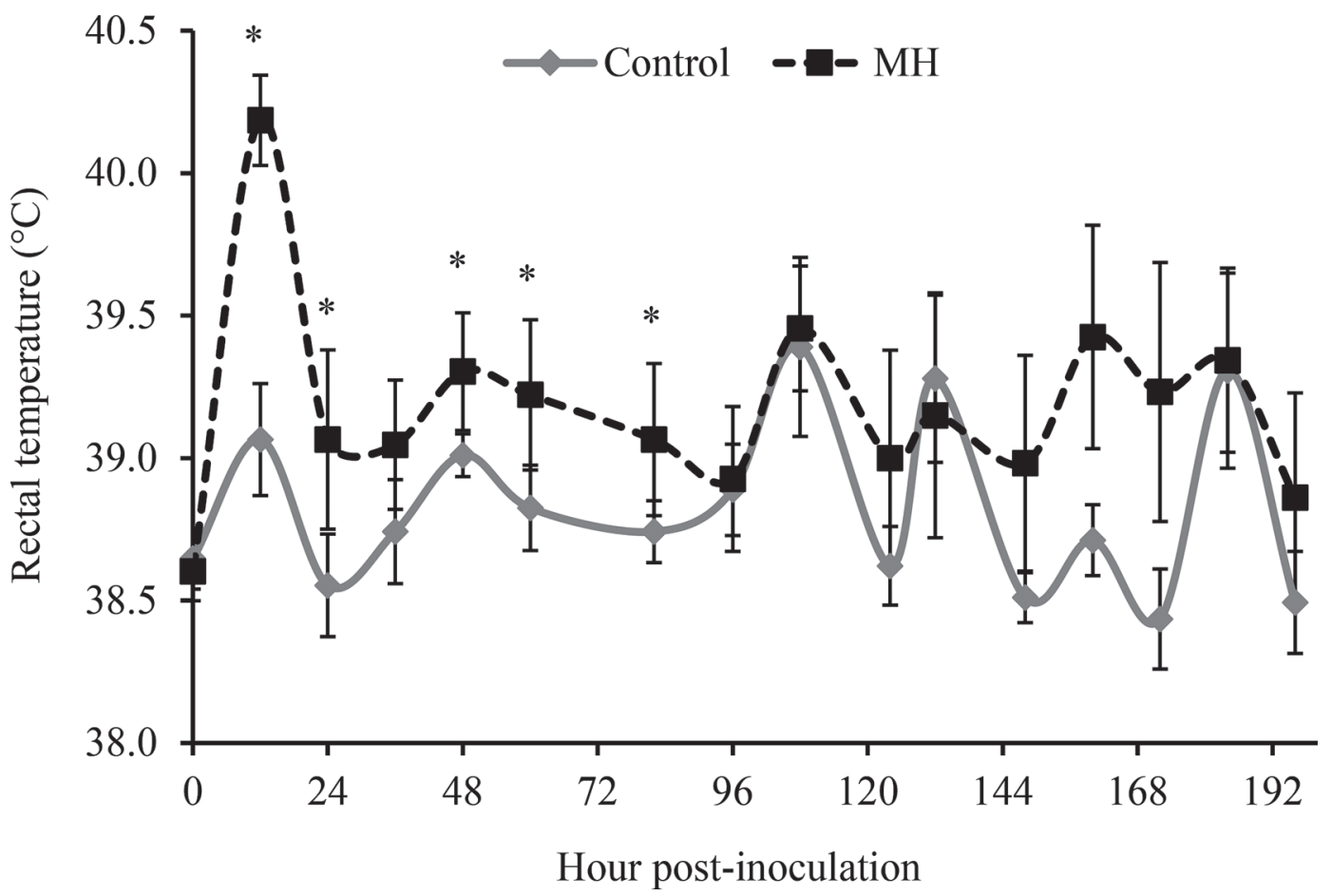

Figure 1. Rectal temperature of calves $(35.9 \pm 8.8 \mathrm{~d}$ of age $)$ housed in groups (6 calves/pen) exposed to a respiratory disease challenge induced by endoscopic inoculation with Mannheimia haemolytica (MH) or inoculated with sterile saline only as control. Temperatures were recorded at 12-h intervals (except for missing data at $\mathrm{h} 72$ ) for $1 \mathrm{wk}$ following exposure to treatment. Values are reported as LSM with error bars denoting SE. Differences by time point denoted as $* P \leq 0.01$. 
by pen interaction $\left(\mathrm{F}_{3,16}=3.64 ; P=0.036\right)$ : in pens of younger calves, $\mathrm{MH}$ calves spent less time overall consuming concentrate $(0.31$ vs. $0.91 \mathrm{~min} / \mathrm{h}, \mathrm{MH}$ vs. $\mathrm{CON}$ in pen $3 ; \mathrm{SE}=0.60 ; \mathrm{F}_{1.16}=6.76 ; P=0.019$, and 0.24 vs. $0.81 \mathrm{~min} / \mathrm{h}, \mathrm{MH}$ vs. $\mathrm{CON}$ in pen $4 ; \mathrm{SE}=0.16$; $\left.\mathrm{F}_{1,16}=7.11 ; P=0.017\right)$ whereas there was no effect of treatment on time spent consuming concentrate in pens of older calves $(P>0.32)$.

Activity and social resting behavior responded to the disease challenge. Lying time during the 3 -d period postinoculation was subject to a treatment by day interaction $\left(\mathrm{F}_{2,40}=11.26 ; P<0.001\right)$, with $\mathrm{MH}$ calves spending more time lying on $\mathrm{d} 0$, but slowly decreasing lying time on subsequent days (Figure 3a). Lying time was also subject to a pen effect $\left(\mathrm{F}_{3,14}=6.61 ; P\right.$ $=0.0052$ ), with pens of younger calves generally lying for longer overall. Lying bout frequency was less influenced by treatment, but tended to differ between treatments over time $\left(\mathrm{SE}=0.12 ; \mathrm{F}_{2,40}=2.47 ; P=\right.$ 0.097), increasing over time for $\mathrm{MH}$ calves to a greater extent than control. The $\mathrm{MH}$ calves were inactive for a greater proportion of their standing time overall across the 3 -d observation period ( 4.20 vs. $2.08 \%$; $\mathrm{SE}=0.91$; $\mathrm{F}_{1,14}=6.66 ; P=0.022$ ). Duration of social lying time did not differ between treatments over time (31.08 vs. $\left.29.8 \mathrm{~min} / \mathrm{h} ; \mathrm{SE}=1.6 ; \mathrm{F}_{2.44}=2.16 ; P=0.13\right)$ but social lying bout frequency was subject to a treatment by day interaction $\left(\mathrm{F}_{2,44}=5.93 ; P=0.0053\right)$, decreasing for $\mathrm{MH}$ calves on $\mathrm{d} 0$ and increasing over time relative to control calves (Figure 3b).

Lying laterality differed between treatments (treatment by side interaction; $\mathrm{F}_{1,20}=4.40 ; P=0.048$ ), with $\mathrm{MH}$ calves lying for longer on their left than their right side (604 vs. $471 \mathrm{~min} / \mathrm{d}$; left vs. right side; $\mathrm{SE}=32 ; \mathrm{t}$ $=2.90 ; P=0.0082)$ over the 3 -d following treatment. Control calves expressed no lateral preference (526 vs. $522 \mathrm{~min} / \mathrm{d}$; left vs. right side; $\mathrm{SE}=30 ; \mathrm{t}=0.10 ; P=$ $0.92)$.

Grooming and active social behaviors were also influenced by the disease challenge. Frequency of selfgrooming was subject to a treatment by day interaction $\left(\mathrm{F}_{2,44}=13.5 ; P<0.001\right)$, with $\mathrm{MH}$ calves engaging in fewer self-grooming bouts on d 0 and increasing frequency over time (Figure 3c). Self-grooming duration followed a similar pattern to self-grooming frequency and was subject to a treatment by day interaction $\left(\mathrm{F}_{2,44}\right.$ $=3.77 ; P=0.03)$, with $\mathrm{MH}$ calves spending less time self-grooming on d $0(0.05$ vs. $2.1 \mathrm{~min} / \mathrm{h} ; \mathrm{MH}$ vs. CON; $\left.\mathrm{SE}=0.42 ; \mathrm{F}_{1,44}=11.6 ; P=0.001\right)$ and $\mathrm{d} 1$ (1.33 vs. $2.50 \mathrm{~min} / \mathrm{h} ; \mathrm{MH}$ vs. $\mathrm{CON} ; \mathrm{SE}=0.42 ; \mathrm{F}_{1,44}=3.89$; $P=0.05)$ but returning to baseline on $\mathrm{d} 2$ (2.88 vs. $\left.3.40 \mathrm{~min} / \mathrm{h} ; \mathrm{SE}=0.42 ; \mathrm{F}_{1.44}=0.75 ; P=0.39\right)$. The frequency of receiving social grooming did not differ between treatments over time (4.74 vs. 4.16 no./h; $\mathrm{MH}$

\section{Control $\square$ Calves inoculated with $\mathrm{MH}$}
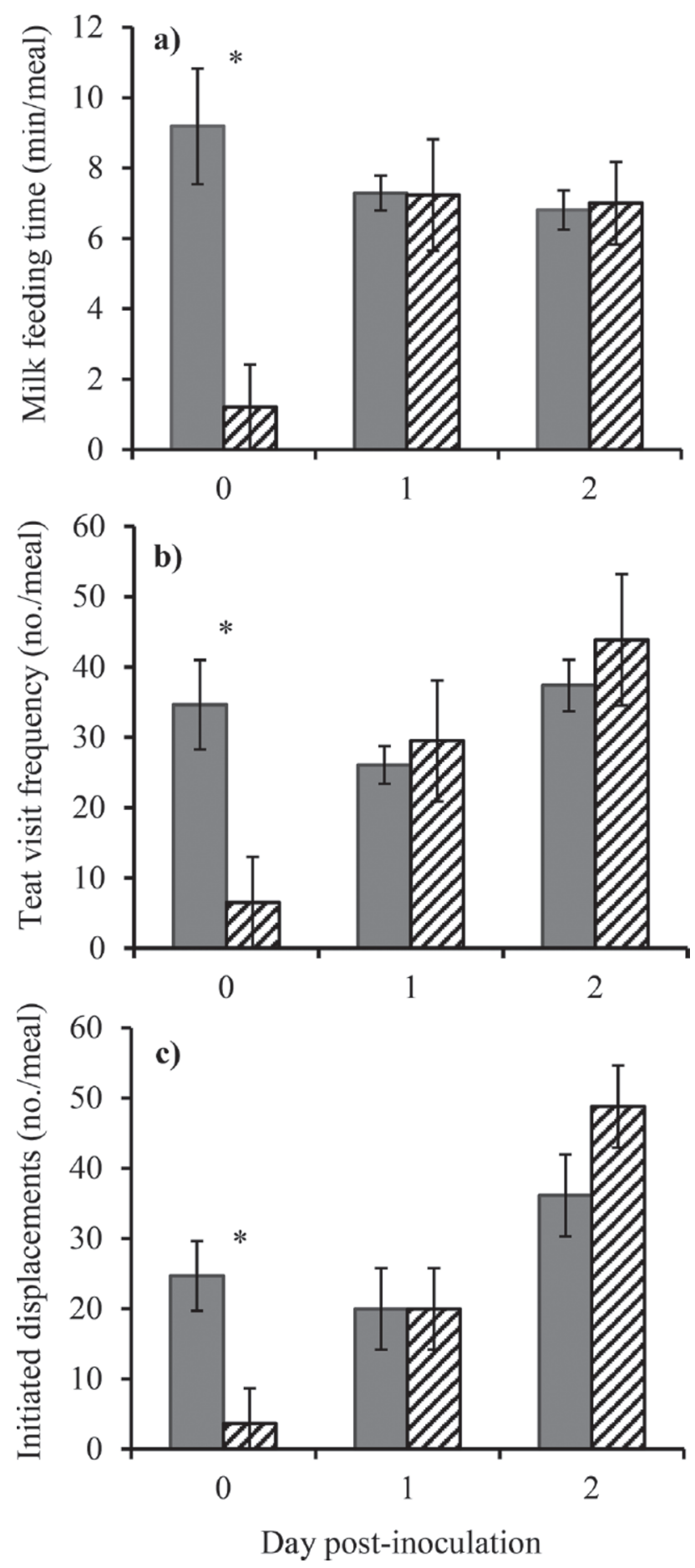

Figure 2. Effects of a respiratory disease challenge induced by endoscopic inoculation with Mannheimia haemolytica (MH) compared with inoculation with sterile saline only (control) on feeding behavior outcomes of calves (35.9 $\pm 8.8 \mathrm{~d}$ of age) housed in groups (6 calves/ pen) for $3 \mathrm{~d}$ following inoculation: (a) milk feeding time (min/meal of milk delivered; $8 \mathrm{~L} / \mathrm{d}, 2 \times / \mathrm{d}$ ), (b) frequency of visiting the teat (no./ meal), and (c) initiated displacements (no./meal). Values are reported as LSM with error bars denoting SE. Differences by time point denoted as $* P \leq 0.01$. 


\section{Control $\square$ Calves inoculated with MH}
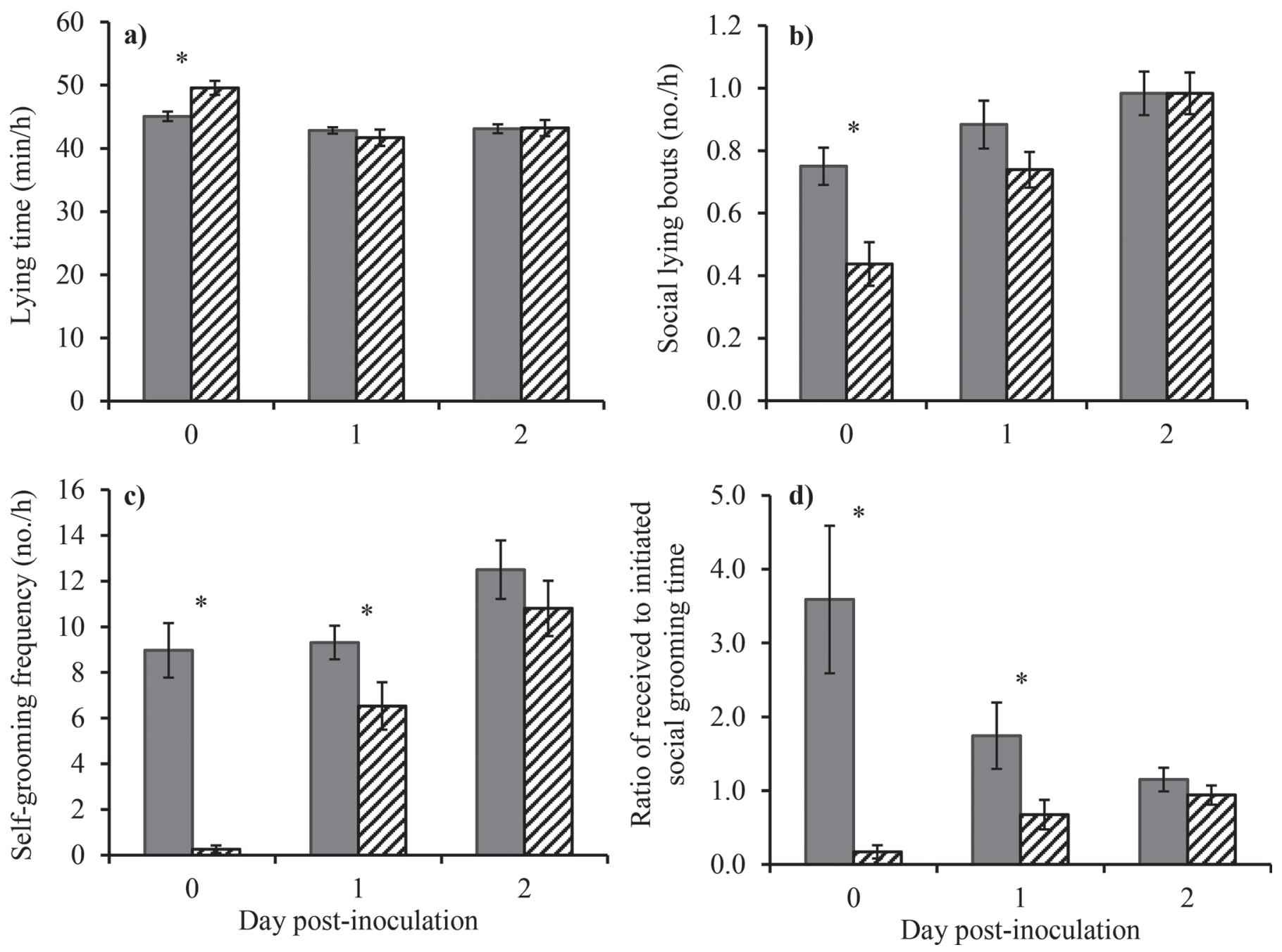

Figure 3. Effects of a respiratory disease challenge induced by endoscopic inoculation with Mannheimia haemolytica (MH) compared with inoculation with sterile saline only (control) on (a) lying time $(\mathrm{min} / \mathrm{h}),(\mathrm{b})$ frequency of social lying bouts (no./h), (c) frequency of self-grooming (no./h), and (d) ratio of received to initiated social grooming time of calves (35.9 $\pm 8.8 \mathrm{~d}$ of age) housed in groups (6 calves/pen) for 3 -d postinoculation. Values are reported as LSM with error bars denoting SE. Differences by time point denoted as ${ }^{*} P \leq 0.01$.

vs. $\left.\mathrm{CON} ; \mathrm{SE}=0.39 ; \mathrm{F}_{2,44}=0.78 ; P=0.46\right)$, but $\mathrm{MH}$ calves tended to receive social grooming for longer periods of time overall $(1.59$ vs. $1.25 \mathrm{~min} / \mathrm{h} ; \mathrm{SE}=0.13$; $\left.\mathrm{F}_{1,16}=3.62 ; P=0.075\right)$. The $\mathrm{MH}$ calves tended to initiate social grooming less frequently overall (3.36 vs. 4.68 no. $\left./ \mathrm{h} ; \mathrm{SE}=0.49 ; \mathrm{F}_{1,16}=3.24 ; P=0.090\right)$ and spent less time grooming another calf on d 0 (0.78 vs. $\left.1.96 \mathrm{~min} / \mathrm{h} ; \mathrm{SE}=0.38 ; \mathrm{F}_{1,44}=4.8 ; P=0.033\right)$. The ratio of received to initiated social grooming time was subject to a treatment by day effect $\left(\mathrm{F}_{2,44}=3.65 ; \mathrm{P}\right.$ $=0.034$ ), occurring less frequently in $\mathrm{MH}$ calves on $\mathrm{d}$ 0 and increasing over time for $\mathrm{MH}$ calves relative to control calves (Figure 3d). Head butting was observed infrequently on only d 1 and 2 postinoculation, and was subject to a treatment by day interaction $\left(\mathrm{F}_{1,11}=7.10\right.$;
$P=0.022$ ), occurring less frequently in $\mathrm{MH}$ calves on $\mathrm{d}$ 1 (0.19 vs. 0.71 no. $/ \mathrm{h} ; \mathrm{MH}$ vs. $\mathrm{CON} ; \mathrm{SE}=0.075 ; \mathrm{F}_{1,11}$ $=15.97 ; P=0.0021)$ with no difference observed on $\mathrm{d}$ $2\left(\mathrm{~F}_{1,11}=0.07 ; P=0.79\right)$.

Minimal differences were observed between treatments in response to a novel object test conducted 2 $\mathrm{d}$ following inoculation. Control and $\mathrm{MH}$ calves did not differ in their latency to touch the novel object (4.05 vs. $3.13 \mathrm{~min} ; \mathrm{SE}=1.0 ; \mathrm{F}_{1,16}=0.42 ; P=0.53$ ) and duration of time in contact with the ball (1.04 vs. $\left.0.94 \mathrm{~min} ; \mathrm{SE}=0.21 ; \mathrm{F}_{1,16}=0.11 ; P=0.74\right)$. However, $\mathrm{MH}$ calves spent less time in close proximity (within a half-head length) to the ball (0.58 vs. $1.65 \mathrm{~min} ; \mathrm{SE}=$ $\left.0.22 ; \mathrm{F}_{1,16}=12.5 ; P=0.002\right)$. Although not affected by treatment, latency to touch the ball and duration of 
time in contact with the ball were both subject to a pen effect $(P<0.005)$, with older calves generally having lower latency to touch the ball and spending more time in contact with the ball.

\section{DISCUSSION}

In this study, we aimed to characterize components of sickness behavior in group-housed calves, utilizing an experimental disease challenge model. As expected, inoculation with $M$. haemolytica resulted in slight, but broad effects on feeding, activity, and social interactions.

With this disease model, we intended to produce a mild disease state, as identifying cases of mild disease is expected to be more challenging than identifying calves experiencing severe disease. The changes we observe in rectal temperatures and health scores suggest that the experimental disease challenge model resulted in a mild disease state. Consistent with results reported by Eberhart et al. (2017) using this challenge model, rectal temperatures of $\mathrm{MH}$ calves peaked at 12-h following inoculation. Although the peak temperature was numerically lower in the present study (40.1 vs. $41.3^{\circ} \mathrm{C}$ at $12 \mathrm{~h}$ postinoculation; present study vs. Eberhart et al., 2017), we found that rectal temperatures were subject to an overall treatment effect, remaining elevated for $\mathrm{MH}$ calves over the study period (Figure 1). In contrast, Eberhart et al. (2017) reported that rectal temperatures of inoculated calves promptly returned to baseline. The continued elevation of rectal temperatures in the present study suggests that this model mirrors naturally occurring respiratory disease progression, in which increased temperatures persist (Zecchinon et al., 2005).

We found that infected calves spent more time lying and standing inactive. This increase in resting coinciding with disease is consistent with previous findings in dairy calves (Theurer et al., 2013) and cows (Chapinal et al., 2010), as well as a range of other species (e.g., primates; Ghai et al., 2015). The increased time standing inactive is consistent with results reported by Borderas et al. (2008), who characterized sickness behavior associated with an LPS challenge model. Inactive standing could be interpreted as stoicism, with calves attempting to mask vulnerability (Weary et al., 2009). In group-housed animals, we can also speculate that standing is socially facilitated to some degree, but that sick calves might be less likely to engage in another activity once standing. We encourage further research evaluating associations between inactive standing and health status.

Interestingly, we found that inoculated calves spent more time lying on their left side than their right. Pre- vious research utilizing a similar pathogen challenge model (Eberhart et al., 2017) inoculated calves in the right lung lobe and found that infected calves spent more time lying on their right side than their left. In contrast, we did not predict differences in lying laterality, as we inoculated calves at the tracheal bifurcation, which should have allowed for a relatively even distribution of the inoculum across both lungs. It is possible that the changes in lying laterality we observed relate to anatomical differences between the right and left lungs: the arterial bronchus of the right lung is larger than that of the left and supplies a greater portion of the lung (Stamp, 1948). Further, there is some evidence of laterality in naturally occurring cases of respiratory disease. Epperson (2003) found that cattle with lung lesions affecting only a single lung were more likely to be affected in the right lung (75.7\%). Although we were not able to characterize the location and severity of lung lesions in the present study, the differences in lying laterality we observed may be explained by the greater potential for lesions in the right lung, with infected calves potentially shifting lying side due to painassociated discomfort. We encourage further research to evaluate changes in lying laterality associated with lung lesions in naturally occurring bouts of respiratory disease or other situations of discomfort.

As expected, we observed changes in feeding time and competitive interactions surrounding feeding in response to the disease challenge. We found that $\mathrm{MH}$ calves spent less time at the teat when milk was available. In previous studies, effects of disease on milk intake depend on milk allowance; calves provided greater quantities of milk or milk replacer have increased visit duration and reduced milk intake and visit frequency to an automated feeder when sick (calves provided $12 \mathrm{~L} / \mathrm{d}$ or ad libitum access to milk or milk replacer; Borderas et al., 2009), but behavioral responses are more subtle and less consistent when milk allotment is restricted: Svensson and Jensen (2007) described a tendency for less frequent visits to an automated milk feeder in sick calves provided 5.6 to $8.1 \mathrm{~L} / \mathrm{d}$, and Borderas et al. (2009) described shorter meal durations but no changes in visit frequency in sick calves provided $4 \mathrm{~L} / \mathrm{d}$. Similar to observations in sick rats (Cohn and de Sá-Rocha, 2006), MH calves were engaged in fewer competitive displacements surrounding milk feeding, as either the actor or reactor, following the challenge. This suggests that sick calves may be likely to avoid feeding at peak times in social feeding environments. Whereas Svensson and Jensen (2007) reported that sick calves had less frequent unrewarded visits to the milk feeder, we observed no effect of disease on frequency of nonnutritive sucking, although we found that inoculated calves spent less time sucking during nonnutritive visits. Disease has 
previously been associated with reductions in feeding time in older cattle. For example, Sowell et al. (1998) found that healthy steers spent more time at the feed bunk and had lower latency to begin feeding compared with sick steers. We found that time spent consuming concentrate decreased following inoculation in pens of younger calves, suggesting that they may have been less motivated to consume solid feed.

It has been suggested that behaviors that are less critical for survival and primarily support long-term fitness are most affected by disease (Weary et al., 2009). For example, play, which primarily supports an animal's longer-term fitness (Špinka et al., 2001), is decreased in calves following disbudding (Mintline et al., 2013), and may also be expected to decrease in response to disease. We found that $\mathrm{MH}$ calves engaged in less head butting, which was generally associated with play. Similar to other observations in sick calves (Borderas et al., 2008) and cows (Fogsgaard et al., 2012), MH calves also had less frequent and shorter incidences of self-grooming. Similar response in self-grooming are seen in a broad range of species, including primates (Ghai et al., 2015).

As predicted, we found that $\mathrm{MH}$ calves exhibited some changes in social behavior and were less likely to initiate social grooming. Barbe et al. (2016) also described reduced active social interactions, including sniffing and grooming, in a rat model of voluntary overwork leading to increased inflammatory cytokines. Reductions in social interactions may be indicative of either general lethargy or a motivation for social isolation during disease. Proudfoot et al. (2014) described isolation behavior, increased time spent in secluded areas, in cows with elevated rectal temperatures and some signs of infection (mastitis, metritis, or pneumonia) during the period immediately postcalving. Social withdrawal has also been observed in sick rodents (Arakawa et al., 2010). It has been proposed that social withdrawal associated with disease in gregarious animals may have an evolutionary function to decrease disease transmission (Loehle, 1995). Interestingly, although infected calves initiated fewer social grooming interactions, they tended to receive a greater duration of social grooming, and the duration of social lying did not differ between treatments. This suggests that healthy calves did not avoid their infected penmates. This is consistent with evidence that healthy rodents did not avoid social contact with familiar, sick animals and engaged equally with both sick and healthy animals (Arakawa et al., 2010). Despite evidence of social withdrawal in sick animals, evidence also indicates that social interaction is beneficial to an animal's recovery from a stressful event (Kikusui et al., 2006); for example, social contact has been shown to reduce stroke-related neurological damage in rats (Hattori et al., 2000). We encourage further research to explore preference and motivation for social withdrawal in sick calves, and the effects of social contact on disease recovery.

In addition to behavioral observations, we conducted a novel object test within the group home pen to assess changes in exploratory behavior associated with disease, but found no difference between treatments in latency to touch and duration of time in contact with a novel object. However, we did observe that $\mathrm{MH}$ calves spent less time in close proximity to the ball (described as "almost touching"; within a half head-length), which may be interpreted as decreased exploratory behavior. A greater difference in response may have been expected had the novel object test been conducted earlier; in the time frame that the test was conducted $(48 \mathrm{~h}$ postchallenge) many other behavioral differences between treatments had diminished (e.g., see Figures 2 and 3 ). It is also possible that calves may have responded differently if we had tested them individually, as the presence of social companions may influence exploratory behavior (Boissy and Le Neindre, 1990; Munksgaard and Jensen, 1996). However, Cramer and Stanton (2015) used a similar novel object test and found that calves with clinical signs of bovine respiratory disease were less likely to approach a novel object or stationary human compared with healthy calves, suggesting that there may be some potential to use group level behavioral tests to assess calf health status.

\section{CONCLUSIONS}

We found that calves exposed to Mannheimia haemolytica in an experimental disease challenge model showed clinical signs of a mild disease state and had a range of altered behavioral patterns compared with healthy calves within the same pen. The findings from this study suggest that a broad range of behavioral responses, including changes in activity, feeding, and direction of social interactions, may be useful indicators of early stages of disease in group-housed calves. Further work is needed to better identify the key indicators of disease and determine the potential for these behavioral indicators to help identify sick calves on-farm.

\section{ACKNOWLEDGMENTS}

We thank the staff and students at the Dairy Research Unit of the University of Florida. In particular, we thank Rachel Brick, Amber DeWitt, Codie Whitehead, James Gross, and McKenzie Holmes for their technical assistance. This project was funded through University of Florida Institute of Food and Agricultural Sciences (Gainesville, FL) Early Career Scientist Seed Funding. This work was supported by the USDA Na- 
tional Institute of Food and Agriculture, Hatch project 1005725 .

\section{REFERENCES}

Arakawa, H., K. Arakawa, and T. Deak. 2010. Sickness-related odor communication signals as determinants of social behavior in rat: A role for inflammatory processes. Horm. Behav. 57:330-341.

Barbe, M. F., D. L. Xin, J. Hadrévi, M. E. Elliot, and A. E. BarrGillespie. 2016. Sickness behaviors (reduced social interaction and pain behaviors) are linked to inflammatory mechanisms in a rat model of work-related musculoskeletal disorders. Proc. Hum. Factors Ergonom. Soc. 60:975-979.

Boissy, A., and P. Le Neindre. 1990. Social influences on the reactivity of heifers: Implications for learning abilities in operant conditioning. Appl. Anim. Behav. Sci. 25:149-165.

Bonk, S., O. Burfeind, V. S. Suthar, and W. Heuwieser. 2013. Technical note: Evaluation of data loggers for measuring lying behavior in dairy calves. J. Dairy Sci. 96:3265-3271.

Borderas, T. F., A. M. De Passillé, and J. Rushen. 2008. Behavior of dairy calves after a low dose of bacterial endotoxin. J. Anim. Sci. $86: 2920-2927$.

Borderas, T. F., J. Rushen, M. A. G. von Keyserlingk, and M. B. de Passillé. 2009. Automated measurement of changes in feeding behavior of milk-fed calves associated with illness. J. Dairy Sci. 92:4549-4554.

Chapinal, N., A. M. de Passillé, J. Rushen, and S. Wagner. 2010. Automated methods for detecting lameness and measuring analgesia in dairy cattle. J. Dairy Sci. 93:2007-2013.

Cohn, D. W. H., and L. C. de Sá-Rocha. 2006. Differential effects of lipopolysaccharide in the social behavior of dominant and submissive mice. Physiol. Behav. 87:932-937.

Cramer, M. C., and A. L. Stanton. 2015. Associations between health status and the probability of approaching a novel object or stationary human in preweaned group-housed dairy calves. J. Dairy Sci. 98:7298-7308.

De Paula Vieira, A., M. A. G. von Keyserlingk, and D. M. Weary 2010. Effects of pair versus single housing on performance and behavior of dairy calves before and after weaning from milk. J. Dairy Sci. 93:3079-3085.

Duve, L. R., and M. B. Jensen. 2012. Social behavior of young dairy calves housed with limited or full social contact with a peer. J. Dairy Sci. 95:5936-5945.

Eberhart, N., J. M. Storer, M. Caldwell, A. M. Saxton, and P. D. Krawczel. 2017. Behavioral and physiologic changes in Holstein steers experimentally infected with Mannheimia haemolytica. Am. J. Vet. Res. 78:1056-1064.

Epperson, W. B. 2003. A preliminary assessment of lung lesion distribution in fed cattle. Page 15 in South Dakota Beef Report, Volume 4. Department of Animal Science of South Dakota State University. South Dakota State University, Brookings.

Færevik, G., I. L. Andersen, M. B. Jensen, and K. E. Bøe. 2007. Increased group size reduces conflicts and strengthens the preference for familiar group mates after regrouping of weaned dairy calves (Bos taurus). Appl. Anim. Behav. Sci. 108:215-228.

Fogsgaard,, K. K., C. M. Røntved, P. Sørensen, and M. S. Herskin. 2012. Sickness behavior in dairy cows during Escherichia coli mastitis. J. Dairy Sci. 95:630-638.

Friard, O., and M. Gamba. 2016. BORIS: A free, versatile open-source event-logging software for video/audio coding and live observations. Methods Ecol. Evol. 7:1325-1330.

Ghai, R. R., V. Fugère, C. A. Chapman, T. L. Goldberg, and T. J Davies. 2015. Sickness behaviour associated with non-lethal infections in wild primates. Proc. Biol. Sci. 282. https://doi.org/10 1098/rspb.2015.1436.

Griffin, D., M. M. Chengappa, J. Kuszak, and D. S. McVey. 2010. Bacterial pathogens of the bovine respiratory disease complex. Vet. Clin. North Am. Food Anim. Pract. 26:381-394.

Hart, B. L. 1988. Biological basis of the behavior of sick animals. Neurosci. Biobehav. Rev. 12:123-137.
Hattori, K., H. Lee, P. D. Hurn, B. J. Crain, R. J. Traystman, and A C. DeVries. 2000. Cognitive deficits after focal cerebral ischemia in mice. Stroke 31:1939-1944.

Kelly, A. P., and E. D. Janzen. 1986. A review of morbidity and mortality rates and disease occurrence in North American feedlot cattle. Can. Vet. J. 27:496-500.

Kikusui, T., J. T. Winslow, and Y. Mori. 2006. Social buffering: Relief from stress and anxiety. Philos. Trans. R. Soc. B Biol. Sci $361: 2215-2228$

Loehle, C. 1995. Social barriers to pathogen transmission in wild animal populations. Ecology 76:326-335.

Love, W. J., T. W. Lehenbauer, P. H. Kass, A. L. Van Eenennaam, and S. S. Aly. 2014. Development of a novel clinical scoring system for on-farm diagnosis of bovine respiratory disease in pre-weaned dairy calves. PeerJ 2:e238.

Miller-Cushon, E. K., R. Bergeron, K. E. Leslie, G. J. Mason, and T. J. DeVries. 2014. Competition during the milk-feeding stage influences the development of feeding behavior of pair-housed dairy calves. J. Dairy Sci. 97:6450-6462.

Mintline, E. M., M. Stewart, A. R. Rogers, N. R. Cox, G. A. Verkerk, J. M. Stookey, J. R. Webster, and C. B. Tucker. 2013. Play behavior as an indicator of animal welfare: Disbudding in dairy calves. Appl. Anim. Behav. Sci. 144:22-30.

Munksgaard, L., and M. B. Jensen. 1996. The use of "open field" tests in the assessment of welfare of cattle. Acta Agric. Scand. A Anim. Sci. (Suppl. 27):82-85

Proudfoot, K. L., M. B. Jensen, D. M. Weary, and M. A. G. von Keyserlingk. 2014. Dairy cows seek isolation at calving and when ill. J. Dairy Sci. 97:2731-2739.

Sowell, B. F., J. G. P. Bowman, M. E. Branine, and M. E. Hubbert. 1998. Radio frequency technology to measure feeding behavior and health of feedlot steers. Appl. Anim. Behav. Sci. 59:277-284.

Sowell, B. F., M. E. Branine, J. G. P. Bowman, M. E. Hubbert, H W. Sherwood, and W. F. Quimby. 1999. Feeding and watering behavior of healthy and morbid steers in a commercial feedlot. J. Anim. Sci. 77:1105-1112.

Špinka, M., R. C. Newberry, and M. Bekoff. 2001. Mammalian play: Training for the unexpected. Q. Rev. Biol. 76:141-168.

Stamp, J. T. 1948. The distribution of the bronchial tree in the bovine lung. J. Comp. Pathol. Ther. 58:1-8.

Svensson. C., and M. B. Jensen. 2007. Short communication: Identification of diseased calves by use of data from automatic milk feeders. J. Dairy Sci. 90:994-997.

Svensson, C., and P. Liberg. 2006. The effect of group size on health and growth rate of Swedish dairy calves housed in pens with automatic milk-feeders. Prev. Vet. Med. 73:43-53.

Svensson, C., K. Lundborg, U. Emanuelson, and S. O. Olsson. 2003 Morbidity in Swedish dairy calves from birth to 90 days of age and individual calf-level risk factors for infectious diseases. Prev. Vet. Med. 58:179-197.

Theurer, M. E., D. E. Anderson, B. J. White, M. D. Miesner, D. A. Mosier, J. F. Coetzee, J. Lakritz, and D. E. Amrine. 2013. Effect of Mannheimia haemolytica pneumonia on behavior and physiologic responses of calves during high ambient environmental temperatures. J. Anim. Sci. 91:3917-3929.

UBC AWP. 2013. UBC Animal Welfare Program: SOP - HOBO Data Loggers. University of British Columbia, Vancouver, Canada.

USDA. 2008. Part II: Changes in the U.S. Dairy Cattle Industry, 19912007. In NAHMS, ed. Dairy 2007. USDA-APHIS-VS, CEAH, Fort Collins, CO

Veissier, I., V. Gesmier, P. Le Neindre, J. Y. Gautier, and G. Bertrand 1994. The effects of rearing in individual crates on subsequent social behaviour of veal calves. Appl. Anim. Behav. Sci. 41:199-210.

Weary, D. M., J. M. Huzzey, and M. A. G. von Keyserlingk. 2009. Board-invited review: Using behavior to predict and identify il health in animals. J. Anim. Sci. 87:770-777.

Zecchinon, L., T. Fett, and D. Desmecht. 2005. How Mannheimia haemolytica defeats host defence through a kiss of death mechanism. Vet. Res. 36:133-156. 